\title{
Growth rate of surface homeomorphisms and flow equivalence
}

\author{
DAVID FRIED \\ Mathematics Department, Boston University, Boston, Mass. 02215, USA
}

(Received 21 February 1985)

\begin{abstract}
We study which algebraic integers $\lambda \geq 1$ arise as the growth rate of a mapping class of a surface and give conditions that are necessary and perhaps sufficient. Flow equivalence and twisted Lefschetz zeta functions are used to generate families of $\lambda$ 's. Examples and open problems are included.
\end{abstract}

\section{Introduction}

One can associate to a homeomorphism $h: \Sigma \triangleright$ of a compact surface $\Sigma$ a number $\lambda \geq 1$ that measures the exponential rate at which elements of the fundamental group $\pi_{1} \Sigma$ can grow under the action of $h$. From Thurston's work we know that $\lambda$ is an algebraic integer with certain special properties [T1]. These are developed in $\S 1$ where we ask whether the set of $\lambda$ 's is characterized by its known properties.

$\$ 2$ develops the machinery of flow equivalence and zeta functions that we will use to generate large families of surface homeomorphisms and to calculate their corresponding growth rates. We give examples in $\S \S 5,6$ to show that the numbers $\lambda$ are not constrained algebraically in ways one might expect. In particular $\lambda$ can have odd degree or $\lambda$ can have conjugates on the unit circle. Odd degree examples were also found by Arnoux and Yoccoz [AY] using interval exchange maps. So they passed to one-dimensional maps whereas we use three-dimensional flows.

$\$ 3$ presents the twisted zeta function for a map of a graph. In $\$ 4$ we indicate how blowing up points can greatly increase the size of a flow equivalence class and we relate our methods to the problem of classifying Anosov flows. $\S 7$ contains more material about flow equivalence including a 'flow-free' description of it and a generalization to other group actions.

In $\S \S 1,7$ we raise questions concerning the behaviour of growth rates in analogous situations involving higher dimensional spaces or abstract groups. We include in $\$ 1$ the first example of a finitely presented group with an endomorphism whose growth rate is not an algebraic integer.

\section{Growth rates}

Before defining growth rates for surface homeomorphisms we must recall some geometric group theory [FS].

Let $G$ be a finitely generated group and $S$ a finite set of generators for $G$. Given $g \in G$ one calls the shortest length of a factorization of $g$ as a product of terms 
$s^{ \pm 1}, s \in S$, the word length $l_{S}(g)$. If $\alpha: G \rightarrow G$ is a homomorphism then there is an $M \geq 1$ such that:

(*) the numbers $l_{S}\left(\alpha^{p} g\right) /\left(l_{S}(g) M^{p}\right)$ are bounded, over all $g \in G, p \geq 1$.

For instance the maximum length of the image of a generator clearly suffices. We define the growth rate $\lambda(\alpha)$ to be the infimum of the $M$ 's such that (*) holds (many authors refer to $\log \lambda$ as the growth rate).

If another finite generating set $S^{\prime}$ is used then there is a $C>0$ so that $l_{S^{\prime}}(s) \leq C$, $l_{S}\left(s^{\prime}\right) \leq C$, for all $s^{\prime} \in S^{\prime}, s \in S$. One sees that the numbers $l_{S^{\prime}}(g) / l_{s}(g), g \in G$, are bounded away from 0 and $\infty$. So condition $(*)$ is independent of the choice of finite generating set and the growth rate $\lambda$ depends on $\alpha$ alone.

Example 1. Let $G$ be free abelian on generators $s_{1}, \ldots, s_{n}$. Then for $S=$ $\left\{s_{1}, \ldots, s_{n}\right\}, l_{S}(g)$ is the 1 -norm of $g$. If $\alpha: G \rightarrow G$ is a homomorphism, $\lambda(\alpha)$ is the spectral radius of $\alpha$.

Example 2. Let $N=\mathbb{Z}\left[y, y^{-1}\right]$ be the integral finite Laurent series in an indeterminate $y$ as an additive group. Let $G$ be the semidirect product of $N$ by an infinite cyclic group $\left\{t^{i} \mid i \in \mathbb{Z}\right\}$ where $t$ conjugates elements of $N$ by the rule $t y^{i} t^{-1}=y^{i+1}$. Clearly $G$ is generated by $S=\{t, 1\}$.

One sees that $l_{S}\left(y^{i}\right)=l_{S}\left(t^{i} \cdot 1 \cdot t^{-i}\right) \leq 2|i|+1$. On the other hand if $n=\Sigma a_{i} y^{i} \in N$, then we can estimate $l_{S}(n)$ from below as follows. We pass to multiplicative notation and write $x_{i}$ for $y^{i}$, so $t x_{i} t^{-1}=x_{i+1}$ and all the $x_{i}$ 's commute. In any factorization of $n=\prod x_{i}^{a_{i}}$

$$
n=t^{b_{1}} x_{0}^{c_{1}} t^{b_{2}} x_{0}^{c_{2}} \cdots
$$

one has $\sum b_{i}=0$ so one can rewrite this as

$$
\begin{aligned}
n & =\left(t^{d_{1}} x_{0}^{c_{1}} t^{-d_{1}}\right)\left(t^{d_{2}} x_{0}^{c_{2}} t^{-d_{2}}\right) \cdots \\
& =\prod_{j} x_{d_{j}}^{c_{j}} .
\end{aligned}
$$

Clearly $\sum\left|a_{i}\right| \leq \sum_{j}\left|c_{j}\right|$. Varying the factorizations of $n$, we find $\sum\left|a_{i}\right| \leq l_{s}(n)$. Putting these upper and lower estimates together, we have

$(* *)\|n\| \leq l_{s}(n) \leq(2 d(n)+1)\|n\|$,

where $d(n)=\sup \left\{|i||| a_{i} \mid \neq 0\right\}$ is a sort of generalized degree and $\|n\|=\sum\left|a_{i}\right|$ is the 1-norm of $n$.

Let $\alpha: G \rightarrow G$ be an endomorphism that fixes $t$ and preserves $N, \alpha(N) \subset N$. Clearly $\lambda(\alpha)=\lim \sup l_{S}\left(\alpha^{p}(1)\right)^{1 / p}$. We see that $\alpha\left(y^{i}\right)=\alpha\left(t^{i} \cdot 1 \cdot t^{-i}\right)=t^{i} \alpha(1) t^{-i}=y^{i} \alpha(1)$ so that $\alpha$ acts on $N$ by multiplication by the fixed element $q=\alpha(1) \in N$. Iterating $\alpha$, we see that $\alpha^{p}(1)$ is just the $p$ th power $q^{p}$ of $q$.

Note that the degrees $d\left(q^{p}\right)$ grow at most linearly in $p$. It follows from (**) that $\lambda(\alpha)=\lim \sup \left\|q^{p}\right\|^{1 / p}$.

To evaluate $\lambda(\alpha)$ more explicitly, we use the following algebraic result (it could also be proved using operator theory).

LEMMA 1. Suppose $P(z)$ is a polynomial over $\mathbb{C}$. Then

$$
\lim \left\|P^{k}\right\|^{1 / k}=\|P\|_{\infty}
$$

where $\|P\|_{\infty}$ is the sup of the values of $P$ on the unit circle. 
Proof of the lemma. The inequality $\geq$ is trivial. For the other inequality, let $f=P^{k}$ in Cauchy's estimate

$$
\frac{1}{n !}\left|f^{(n)}(0)\right| \leq \frac{1}{2 \pi}\|f\|_{\infty}
$$

and sum over $n$. This gives

$$
\left\|P^{k}\right\| \leq \frac{1}{2 \pi}\|f\|_{\infty}^{k} \cdot d\left(P^{k}\right) .
$$

As $d\left(P^{k}\right)$ grows linearly in $k$, one can take $k$ 'th roots and pass to the limit.

So multiplying $q$ by a suitable power of $y$ to get a polynomial $P$, we see that

$$
\lambda(\alpha)=\|q\|_{\infty}, \quad \text { where } q=\alpha(1) .
$$

In example 1 , there is an eigenvalue $u$ of an integer matrix so that $\lambda=\sqrt{u \bar{u}}$. Since the characteristic polynomial of this matrix is monic with integer coefficients, $u$ is an algebraic integer and hence so is $\lambda$.

In example 2, on the other hand, $\lambda$ need not be an algebraic integer. To evaluate $\lambda=\|q\|_{\infty}$, note that at the maximum of $|q|$ on $S^{1}, q^{\prime}(z) z / q(z) \in \mathbb{R}$. We compute this maximum in the case where $q$ is quadratic, say $q(z)=a z^{2}+b z+c, a \neq 0, a, b, c \in \mathbb{Z}$. At an extremum of $|q(z)|$ for $z=x+i y, x \in[-1,1], y^{2}+x^{2}=1$, one finds $y(a b+b c+$ $4 a c x)=0$. At $y=0$ the values of $q$ are integral, so we will take $x_{0}=(-4 a c)^{-1}(a b+b c)$, $c \neq 0$, and choose $a, b, c$ so $\left|x_{0}\right|<1$. One computes

$$
q(z) q(\bar{z})=a^{2}+b^{2}+c^{2}+(2 a b+2 b c) x+2 a c\left(2 x^{2}-1\right) .
$$

In order that $x_{0}$ be a maximum we need $a c<0$. We find

$$
\lambda^{2}=(a-c)^{2}+b^{2}+(-4 a c)^{-1}(a b+b c)^{2} .
$$

So if $|a b+b c|<-4 a c$ and $4 a c \nmid(a b+b c)^{2}, \lambda$ is not an algebraic integer in example 2. For example $q(z)=z^{2}+z-2$ has this property. It is clear from this computation, however, that the $\lambda$ 's of example 2 are algebraic numbers.

This raises the possibility that $\lambda(\alpha)$ is transcendental for some $G, \alpha$. Even with $G$ as in example 2, if one considers $\alpha$ 's with $\alpha(t)=t^{2}$ then it is not clear whether $\lambda(\alpha)$ must be algebraic. On the other hand we pose:

Problem 1. Show that the growth rate $\lambda(\alpha)$ for an endomorphism $\alpha$ of a finitely generated free group $G$ is an algebraic integer.

This ought to be accessible to a direct combinatorial approach. As will shortly be seen, it holds whenever $\alpha$ arises as the action of a homeomorphism of a surface on $\pi_{1}$. In particular it holds for $G$ with $\leq 2$ generators since then any automorphism can be realized on a punctured torus [MKS].

Definition. Let $\Sigma$ be a compact manifold and $h: \Sigma \rightleftharpoons$ a map. Let $\alpha=h_{*}: \pi_{1} \Sigma \rightleftharpoons$ and $\lambda=\lambda(\alpha)$. Then we write $\lambda=\lambda(h)$, the $\pi_{1}$ growth rate of $h$.

We here use the usual convention where $\pi_{1}$ of a disconnected manifold is the direct sum of $\pi_{1}$ of its components. We have also suppressed considerations of basepoint here: for more details to justify this see [FS]. 
Clearly $\lambda(h)$ is a homotopy invariant. Se we may speak of the $\pi_{1}$ growth rate $\lambda(c)$ of an isotopy class of homeomorphisms.

For $\operatorname{dim} \Sigma=2$ and any $c$, Thurston showed that $\lambda(c)$ is an algebraic integer. There are two arguments for this, each based on his theory of pseudo-Anosov homeomorphisms. We must summarize, then, this theory. We begin by defining measured foliations.

Let $\mathscr{L}$ be the line field on $\mathbb{C}$ defined by $\operatorname{Im}(z)=$ constant. Let $k \geq 3$ and consider the inverse image of $\mathscr{L}$ under $z^{k / 2}$. It is a well-defined line field for $z \neq 0$ with a singularity at $z=0$ that we call a prong singularity of order $k$. By blowing up such a prong, one obtains $k$ singular points on the boundary circle which we will also call prong singularities. A measured foliation $(\mathscr{F}, \mu)$ on a compact surface $\Sigma$ is a foliation, possibly with prong singularities, equipped with a holonomy invariant transverse measure that is positive on every transverse interval.

Suppose $\mathscr{F}_{1}, \mathscr{F}_{2}$ are such measured foliations and that they have the same interior singularities. Suppose that the configuration at each interior singular point $p$ is that obtained by pulling back the transverse foliations $\operatorname{Im} z=$ constant and $\operatorname{Re} z=$ constant under $z^{k / 2}, k=k(p) \geq 3$. At regular interior points suppose $\mathscr{F}_{1}, \mathscr{F}_{2}$ are transverse in the ordinary sense. At boundary curves suppose the configuration is like that obtained by blowing up a prong singularity. We then say $\mathscr{F}_{1}, \mathscr{F}_{2}$ are transverse.

Definition. A homeomorphism $h: \Sigma \rightarrow \Sigma$ of a compact surface $\Sigma$ is pseudo-Anosov if there are measured foliations $\mathscr{F}_{1}, \mathscr{F}_{2}$ on $\Sigma$ and a $\lambda \in(1, \infty)$ so that

(1) $\mathscr{F}_{1}, \mathscr{F}_{2}$ are transverse;

(2) each $\mathscr{F}_{i}$ is preserved by $h$;

(3) the transverse measures $\mu_{i}$ scale under $h$ by $\lambda^{ \pm 1}$, namely

$$
h_{*} \mu_{1}=\lambda \mu_{1}, \quad h_{*} \mu_{2}=\lambda^{-1} \mu_{2} .
$$

The archetypal example of such an $h$ is a linear toral automorphism with eigenvalues $\alpha_{1}, \alpha_{2}$ off the unit circle. One chooses $\mathscr{F}_{i}$ parallel to the $\alpha_{i}$ eigendirections of $h$ : these foliations are transverse (in the usual sense, as they have no singularities) and measured (indeed uniquely ergodic). One has $h_{*} \mu_{1}=\left|\alpha_{2}\right| \mu_{2}$ and $h_{*} \mu=\left|\alpha_{1}\right| \mu_{2}$. So, possibly relabelling our foliations, we see that $h$ is pseudo-Anosov.

By considering how curves on $\Sigma$ grow under iteration of $h$, Thurston showed that the expansion constant $\lambda$ is the same as the growth rate. He also showed that a given isotopy class $c$ could be decomposed along a certain system of disjoint closed curves into a disjoint sum of isotopy classes of subsurfaces, each of which is either periodic or pseudo-Anosov. In this decomposition the growth rate of $c$ is the largest of the growth rates of the pieces. Since periodic pieces have growth rate $\lambda=1$, we may as well suppose, when studying $\lambda$, that $h$ is a pseudo-Anosov that cyclically permutes the $p$ components of a given surface. We have the first reduction of our problem.

LEMMA 2. $\lambda>1$ is a growth rate of a surface homeomorphism $\Leftrightarrow$ for some $p>0, \lambda^{p}$ is the expansion constant of a pseudo-Anosov homeomorphism $h$ of a connected surface. 
The backward implication is proved by taking the $p$ 'th root of $h$, i.e. letting $p$ copies of the connected surface by cyclically permuted so that $h$ is induced on each component at the $p$ 'th iterate.

We turn now to the first proof of integrality. There is a Markov partition for $h$ with an associated transition matrix $A$ with non-negative integer entries. The expansion constant $\lambda$ is an eigenvalue of $A$ corresponding to a certain explicit positive eigenvector defined using the transverse measures [FLP]. This shows $\lambda$ is an eigenvalue of an integer matrix, hence integral.

The fact that the entries of $\boldsymbol{A}$ are non-negative gives more. Suppose for simplicity that $\Sigma$ is connected. Then $h$ is topologically mixing and so $A$ is mixing (i.e. some power of $\boldsymbol{A}$ has positive entries). This implies by a result of Perron \& Frobenius that the eigenvalues $\alpha$ of $A, \alpha \neq \lambda$, satisfy $|\alpha|<\lambda$.

The second proof of integrality is simpler. Let us consider the case of a pseudoAnosov $f$ on a connected $\Sigma$ with both the stable and unstable orientations orientable (this orientability can be arranged by passing to a suitable branched cover). Then each transverse measure can be regarded as a closed Alexander 1 -cochain $\omega_{i}$. Since $f^{*} \omega_{i}= \pm \lambda^{ \pm 1} \omega_{i}$, we see that $\pm \lambda, \pm \lambda^{-1}$ are eigenvalues of $f^{*}: H^{1}(M ; \mathbb{R}) \rightleftharpoons$. But as $f^{*}$ preserves the integral cohomology lattice, this shows $\pm \lambda, \pm \lambda^{-1}$ are algebraic integers. We have almost shown:

THEOREM 1. If $\lambda$ is the growth rate of a surface homeomorphism then $\lambda$ is an algebraic unit (i.e. $\lambda$ and $\lambda^{-1}$ are algebraic integers). Some power $\lambda^{p}$ is the expansion constant of a pseudo-Anosov $h$ on a connected surface and so the algebraic conjugates $\alpha$ of $\lambda^{p}$ satisfy

$$
\alpha \in A\left(\lambda^{p}\right)=\left\{\left(\lambda^{p}\right)^{-1} \leq|z| \leq \lambda^{p}\right\},
$$

with at most one conjugate on a given boundary component of this annulus.

Remark. If $\lambda^{-1}$ is algebraically conjugate to $\lambda$ (it isn't always) then this follows from the estimate $|\alpha|<\lambda$ above.

The uniqueness in the lemma depends on the Lefschetz formula and symbolic dynamics. As $\Sigma$ is connected $h$ is topologically mixing, so Bowen's argument shows that \#Fix $\left(h^{n}\right) / \lambda^{n} \rightarrow 1$ as $n \rightarrow \infty$ (cf. the lemma in [F8]). We want to show |Trace $h^{* n} \mid / \lambda^{n} \rightarrow 1$, in order to rule out the existence of other eigenvalues of $h^{*}$ of modulus $\lambda$. It suffices to show that \#Fix $\left(h^{n}\right)$ and $\mid$ Trace $h^{n} \mid$ have a bounded difference.

But $L\left(h^{n}\right)=\Sigma$ ind $\left(h^{n}, p\right)$ by the Lefschetz formula, where $p$ varies over Fix $\left(h^{n}\right)$ and ind denotes the Lefschetz index. The contributions of $H^{\circ}, H^{2}$ to the left hand side and of the singular periodic points to the right hand side are bounded over $n$. Moreover all the nonsingular points in Fix $\left(h^{n}\right)$ have the same fixed point index since $\Sigma$ is connected and the foliations are orientable.

It appears from examples such as those given in $\$ \S 5,6$ below that $\lambda$ satisfies no other algebraic constraints. So we ask:

Problem 2. Suppose $\beta$ is an algebraic unit, $\beta>1$, all algebraic conjugates of $\beta$ lie in the annulus $A(\beta)=\left\{|z| \in\left[\beta^{-1}, \beta\right]\right\}$ and at most one lies on either boundary. Is 
there a power $\beta^{p}, p=1,2, \ldots$ that is the expansion constant of a pseudo-Anosov homeomorphism?

An affirmative answer would solve the problem of which $\lambda$ 's arise as growth rates of surface homeomorphisms.

We summarize this section by saying that growth rates of surface homeomorphisms frequently arise as eigenvalues of the induced maps on first homology.

Remark. If one studies the case $\lambda=1$ more closely, one can assign a polynomial growth rate to a surface homeomorphism with subexponential growth. The polynomial growth is either constant or linear, in fact. This gives examples of free group automorphisms that cannot be represented by surface homeomorphisms [F7]. The constraints on $\lambda$ in theorem 1 give other examples of this sort with $\lambda>1$.

\section{Zeta functions and flow equivalence}

We will develop in this section a method for prodıcing large families of pseudoAnosov expansion constants. Before explaining why the method works we will give an example (developed further in coming sections) of how it works.

Consider the polynomial in two variables

$$
p(\xi, \eta)=1-\left(4+\eta+\eta^{-1}\right) \xi+\xi^{2} .
$$

By substituting $t^{a}$ for $\eta$ and $t^{b}$ for $\xi$ one obtains a family of 'polynomials'

$$
p_{a b}(t)=1-\left(4+t^{a}+t^{-a}\right) t^{b}+t^{2 b}, \quad a, b \in \mathbb{Z} .
$$

Properly speaking, $p_{a b}$ is a finite Laurent series in $t$ as non-negative exponents may occur. However if $|a|<b$ then all terms (other than 1) have positive exponent. We will show that for any $b>|a|$, the largest zero of $p_{a b}(t)$ is a pseudo-Anosov expansion constant.

To explain this, recall that a cross-section to a flow $\varphi$ on a compact 3-manifold $M$ is a compact surface $K$ transverse to the flow that meets every flowline and that relates to $\partial M$ in the usual way: $K$ is transverse to $\partial M$ and $\partial K=K \cap \partial M$. We call $\varphi$ circular if it admits cross-sections. To $K$ there corresponds a homeomorphism $r: K \rightarrow K$ called the monodromy or the return map.

The polynomial $p(\xi, \eta)$ is a zeta function attached to a certain such circular flow $\varphi$. The cross-sections to $\varphi$ (strictly speaking, the isotopy classes of cross-sections) correspond to the pairs $a, b$ with $|a|<b$. Each return map $r_{a b}$ is pseudo-Anosov and preserves the stable and unstable orientations. The polynomial $p_{a b}(t)$ is a factor of the characteristic polynomial of the linear map induced by $r_{a b}$ on first homology. As we saw in $\S 1$, the largest zero of this characteristic polynomial is the expansion constant of $r_{a b}$. So we have, to some extent, explained the above example.

The further explanation involves two things. One is the equivalence relation on surface homeomorphisms defined by $r_{1} \sim r_{2}$ if some circular $\varphi$ has cross-sections $K_{1}, K_{2}$ with return maps respectively equal to $r_{1}, r_{2}$. We say [I] $r_{1}$ and $r_{2}$ are flow equivalent. Many qualitative dynamical properties are preserved by flow equivalence, but quantitative ones vary. The second idea involved is the zeta function of $\varphi$. 
It will turn out that this zeta function explains how Lefschetz numbers vary under flow equivalence.

When studying the action $h_{i}: H_{i}(\Sigma ; \mathbb{Q}) \rightleftharpoons$ of a homeomorphism $h: \Sigma \rightleftharpoons$ of a compact surface on homology it is convenient to use the Lefschetz zeta function defined by

$$
\tilde{\zeta}=\tilde{\zeta}(h, t)=\frac{\operatorname{det}\left(I-t h_{1}\right)}{\operatorname{det}\left(I-t h_{0}\right) \operatorname{det}\left(I-t h_{2}\right)} .
$$

It can be viewed as a rational function of $t$ or, expanding the denominator, as a power series in $t$ with constant term 1 . In fact $\tilde{\zeta}$ is a generating function for the sequence of Lefschetz numbers $L\left(h^{n}\right)$ since

$$
\tilde{\zeta}=\exp \sum \frac{L\left(h^{n}\right)}{n} t^{n}
$$

This formula follows formally from the definition

$$
L\left(h^{n}\right)=\text { Trace } h_{0}^{n}-\text { Trace } h_{1}^{n}+\text { Trace } h_{2}^{n}
$$

and the formal power series expansion for $\log 1-\mu t$ by letting $\mu$ vary over the eigenvalues of the $h_{i}$ 's [Fr].

Before developing the analogous zeta function for circular flows, we will recall how one associates a cohomology class $u_{K}$ to a cross-section $K, u_{K} \in H^{1}(M ; \mathbb{Z})$. We need to associate a number $u_{K}(\delta)$ to each closed loop in an additive way. The rule is to isotope $\delta$ to be transverse to $K$ and then to count the points of intersection with weight of +1 if the loop $\delta$ passes through $K$ in the same direction as the flow $\varphi,-1$ if it passes in the opposite direction. In particular $u_{K}(\delta)>0$ if $\delta$ is a closed orbit of $\varphi$, oriented by $\varphi$ in the obvious way.

We next present the zeta function of a circular flow $\varphi$, assuming that the closed orbits $\gamma$ of $\varphi$ are all isolated. Choosing a prime orbit $\gamma$ and a point $p \in \gamma$ we build a small transverse disc $D$ at $p$ and construct the local return map $r_{\gamma}: D^{\prime} \rightarrow D$ where $D^{\prime}$ is a smaller disc containing $p$ and $r_{\gamma}(q)$ is the first point on the positive trajectory through $q$ that again lies in $D$. The germ of $r_{\gamma}$ at $p$ is determined by $\gamma$ above and we have supposed that $p$ is an isolated fixed point of $r_{\gamma}^{n}$ for all $n \geq 1$. So we can discretize the local topology at $\gamma$ by forming the sequence of Lefschetz indices ind $\left(p, r_{\gamma}^{n}\right) \in \mathbb{Z}$ and the local Lefschetz zeta function

$$
\tilde{\zeta}_{\gamma}(t)=\exp \sum_{n \geq 1} \frac{1}{n} t^{n} \cdot \text { ind }\left(p, r_{\gamma}^{n}\right)
$$

The zeta function of $\varphi$ will be a suitable combination of these $\tilde{\zeta}_{\gamma}$ 's and the homology class $[\gamma]$ of the $\gamma$ 's. Here we will take $[\gamma]$ to be in the group $H=H_{1}(M ; \mathbb{Z}) /$ torsion $=$ image $\left(H_{1}(M ; \mathbb{Z}) \rightarrow H_{1}(M ; \mathbb{Q})\right)$. We choose an integral basis $x_{1}, \ldots, x_{\beta}$ for $H$, which we view as a multiplicative group, and we choose a cross-section $K$ for $\varphi$. Since $u_{K}[\gamma]>0$, the power series $\tilde{\zeta}_{\gamma}([\gamma])$ in the variables $x_{i}^{ \pm 1}$ makes good sense formally. Since each value of $u_{K}[\gamma]$ occurs for only finitely many $\gamma$ (the return map $r_{K}$ has only finitely many points of each period and the period of $\gamma$ for $r_{K}$ is Card $(\gamma \cap K)=$ $\left.u_{K}[\gamma]\right)$ we can formally multiply these terms over all $\gamma$. So we have: 
Definition. The twisted Lefschetz zeta function of $\varphi$ is

$$
\tilde{\zeta}(\varphi)=\prod_{\gamma} \zeta_{\gamma}([\gamma])
$$

where $\gamma$ varies over the prime closed orbits of $\varphi$.

The relationship between the zeta functions of $\varphi$ and its return maps, and hence the behaviour of Lefschetz numbers under flow equivalence, is given by:

Proposition 1 [F4]. Let $a_{i}=u_{K}\left(x_{i}\right), i=1, \ldots, \beta$. Then the substitution $x_{i} \rightarrow t^{a_{i}}$ changes $\tilde{\zeta}(\varphi)$ to $\tilde{\zeta}(r, t)$, where $r$ is the return map for $K$.

The proof of this proposition is easy, and depends only on the Lefschetz formula for the iterates of $r$. One checks simply that each $\gamma$ contributes the same to $\tilde{\zeta}(r, t)$ and to $\left.\tilde{\zeta}(\varphi)\right|_{x_{i}=r^{a}}$. It is now clear that changing the cohomology class $u_{K}$ of the cross-section is liable to change the Lefschetz zeta function of the return map, since the substitution $x_{i} \rightarrow t^{a_{i}}$ will vary. One could also see this from the fact that a given closed orbit $\gamma$ for $\varphi$ contributes to $\tilde{\zeta}(r, t)$ according to its period, but this period is exactly $u_{K}(\gamma)$.

One of the main reasons that these algebraic techniques apply to our problem is that anything flow equivalent to a pseudo-Anosov is also pseudo-Anosov. This was shown in [F2]. It is clear that if $h$ is pseudo-Anosov and preserves stable and unstable orientations then the corresponding stable and unstable foliations of $\varphi$ will be oriented. So on any cross-section $K$ to $\varphi$, the induced foliations will be oriented and the pseudo-Anosov return map $r: K \rightarrow K$ will preserve these orientations. We call a circular flow $\varphi$ pseudo-Anosov if some (hence all) return maps for $\varphi$ are pseudo-Anosov.

We can summarize this situation as follows:

Proposition 2. Let $\varphi$ be a circular pseudo-Anosov flow with oriented stable and unstable foliations. If there is a cross-section $K$ with $u_{K}\left(x_{i}\right)=a_{i}, i=1, \ldots, \beta$, then the largest zero of the rational function $\left.\tilde{\zeta}(\varphi)\right|_{x_{i}=t^{a_{i}}}$ is the expansion constant of the return map $r_{K}: K \rightarrow K$.

In order to apply the proposition, one must be able to construct such $\varphi$ 's and to identify those classes in $H^{1}(M ; \mathbb{Z})$ that are dual to cross-sections. The first problem is easy: any given pseudo-Anosov $h: \Sigma \rightarrow \Sigma$ has a suspension flow $\varphi=\varphi_{h}$ with $h$ as monodromy. One takes $M$ to be the mapping torus $M_{h}$ obtained as the orbit space of $\Sigma \times \mathbb{R}$ under the $\mathbb{Z}$ action generated by $g(h(x), t)=(x, t+1)$. The flow $\varphi$ is induced by translation along the $\mathbb{R}$ factor.

The problem of identifying the cohomology classes of sections was solved in [F1]. It was shown there that the closed orbits of a pseudo-Anosov $\varphi$ determine the cross-sections in the sense that $u \in H^{1}(M ; \mathbb{Z})$ is dual to a cross-section $\Leftrightarrow u(\gamma)>0$ for all closed orbits $\gamma$. This latter condition defines an open cone in $H^{1}(M ; \mathbb{R})$ that we denote $\mathscr{C}_{\varphi}$. Cross-sections determine points in $\mathscr{C}_{\varphi} \cap H^{1}(M ; \mathbb{Z})$ and this sets up a 1-1 correspondence between isotopy classes of cross-sections and such lattice points.

To compute with this scheme, it is necessary to know how to identify $\tilde{\zeta}(\varphi)$ in the case of a suspension flow $\varphi=\varphi_{h}$. The computation of $\tilde{\zeta}(\varphi)$ uses the twisted chains 
of $h: \Sigma \rightarrow \Sigma$, i.e. the chains of a certain covering space $\tilde{\Sigma}$ of $\Sigma$. Here $\tilde{\Sigma}$ is the abelian covering of $\Sigma$ whose deck transformation group $G=G(h)$ is the image of $H_{1}(\Sigma ; \mathbb{Z})$ in $H_{1}\left(M_{h} ; \mathbb{Q}\right)$. By the Wang sequence, $G$ is the torsionfree quotient of the cokernel of $h_{*}-$ id: $H_{1}(\Sigma ; \mathbb{Z}) \rightleftharpoons$, i.e. $G$ is the largest torsionfree abelian quotient of $\pi_{1} \Sigma$ on which $h$ acts trivially. Then $h$ lifts to $\tilde{h}: \tilde{\Sigma} \rightarrow \tilde{\Sigma}$ and $\tilde{h}$ commutes with $G$. Choosing some cell decomposition for $\Sigma$ and lifting it to $\tilde{\Sigma}$, one can deform $\tilde{h}$ equivariantly to a cellular map and form the associated chain maps $F_{i}: C_{i}(\tilde{\Sigma} ; \mathbb{Z}) \rightarrow C_{i}(\tilde{\Sigma} ; \mathbb{Z})$ on the twisted chain complex $C_{*}(\tilde{\Sigma} ; \mathbb{Z})$. These maps $F_{i}$ commute with the free action of $G$ on chains and so the $F_{i}$ 's are morphisms of modules over the group ring $\mathbb{Z} G$. Lifting the cells of $\Sigma$ to $\tilde{\Sigma}$ in some fixed way, and ordering them, we can identify the $F_{i}$ 's with matrices over $\mathbb{Z} G$. We now form the rational function (over $\mathbb{Z} G$ )

$$
\tilde{\zeta}_{G}(\tilde{h}, x)=\prod_{i} \operatorname{det}\left(I-x F_{i}\right)^{(-1)^{i+1}},
$$

which we call the twisted Lefschetz zeta function of $h$ [F5]. Changing the choice of lift $\tilde{h}$ only affects $\tilde{\zeta}_{G}$ by substitutions of the form $x \rightarrow g \cdot x$, where $g \in G$ is fixed. The following proposition explains how to compute $\tilde{\zeta}(\varphi)$ from a cross-section and it shows that $\tilde{\zeta}(\varphi)$ is the power series expansions of a rational function.

Proposition 3. Choose an integral basis $x_{2}, \ldots, x_{\beta}$ for $G$ and extend it to a basis $x_{1}, x_{2}, \ldots, x_{\beta}$ for $H$ so that $u_{K}\left(x_{1}\right)>0$. For a proper choice of the lift $\tilde{h}$ (depending on $\left.x_{1}\right)$

$$
\tilde{\zeta}(\varphi)=\tilde{\zeta}_{G}\left(\tilde{h}, x_{1}\right)
$$

It is clear that $\tilde{\zeta}_{G}$ depends only on the homotopy class of $h$. As we will be considering surfaces with boundary, we will study the special case of maps of 1-complexes in the next section.

It is also valuable to be able to recognize $\mathscr{C}_{\varphi}$ from the readily computable zeta function $\tilde{\zeta}_{G}$. If the stable and unstable foliations are orientable this can be done. One reduces $\tilde{\zeta}_{G}$ to lowest terms, say $\tilde{\zeta}_{G}=P(x) / Q(x)$ where $P$ and $Q$ are polynomials over $\mathbb{Z} G$ with constant term 1 . We define the non-trivial support Supp' $(P) \subset H$ to be those $h \neq 1$ that occur in $P$ with non-zero coefficient. Then by a trivial modification of the argument in $[F 3, \S 3]$ one finds:

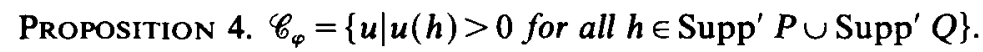

The argument in [F3] was for a circular basic set and twisted Artin-Mazur zeta function, but the finitely many singular orbits have no serious effect and our orientation hypotheses assure that no harmful cancellations take place. So altogether we have shown:

THEOREM 2. Let $h: \Sigma \rightarrow \Sigma$ be a pseudo-Anosov diffeomorphism that preserves stable and unstable orientations. Let $\tilde{\zeta}_{G}(\tilde{h}, x)$ be the twisted Lefschetz zeta function of $h$ and $x_{2}, \ldots, x_{\beta}$ an integral basis for

$$
G=\left(H_{1}(M, \mathbb{Z}) / f_{*} \gamma=\gamma\right) / \text { torsion }
$$

as a multiplicative abelian group. Let $H=\left\{g x^{i} \mid i \in \mathbb{Z}, g \in G\right\}$.

Suppose that in lowest terms $\tilde{\zeta}_{G}(\tilde{h}, x)$ has the form $P(x) / Q(x)$. Then for any homomorphism $u: H \rightarrow \mathbb{Z}$ with $u$ positive on the finite set $\operatorname{Supp}^{\prime}(P) \cup \operatorname{Supp}^{\prime}(Q) \subset H$, 
the substitution $t^{u(h)}$ for $h$ sends $\tilde{\zeta}_{G}(\tilde{h}, x)$ to the Lefschetz zeta function $\tilde{\zeta}(r, t)$ for some pseudo-Anosov $r$ flow equivalent to $x$. The expansion constant $\lambda(r)$ is the largest zero of $\tilde{\zeta}(r, t)$.

Only the last statement needs some amplification. The action of $r$ on the 0 th and 2nd homology has finite order. As $\lambda(r)>1$, it is a zero of the numerator of $\tilde{\zeta}(r, t)$ and not of the denominator.

We have justified the example at the beginning of this section, up to finding an $h$ as in the theorem with $\tilde{\zeta}_{G}=1-\left(4+\eta+\eta^{-1}\right) \xi+\xi^{2}$. Such an $h$ will be found in $\S 4$.

We mention that the $G$ of theorem 1 could be replaced by any abelian quotient of $H_{1}(K ; \mathbb{Z})$ on which $f$ acts and acts trivially. $G$ is the maximal torsionfree group of this sort and so the zeta functions of the entire flow equivalence class can be obtained from $\tilde{\zeta}_{G}$. But intermediate covers are easier to compute with.

\section{The twisted zeta function for a 1-complex}

In this section we will show how to compute the twisted chain maps for a map $f: \Gamma \rightarrow \Gamma$ where $\Gamma$ is a connected finite complex of dimension 1. Such maps arise frequently in the study of surface homeomorphisms, under the guise of branched 1-manifolds or train tracks [T1]. In our examples later on we will take $\Gamma$ to be the 1 -skeleton of a certain cell complex structure on a torus. We will see that the covering space that appears in the definition of $\tilde{\zeta}_{G}$ need not be known explicitly in order to compute it and an algorithm is particularly simple to describe in the 1-dimensional situation.

We suppose, by a preliminary homotopy, that $f$ preserves the set of vertices $V \subset \Gamma, f(V) \subset V$. Then the image of an edge $e$ runs through the edges of $\Gamma$ in some way, beginning and ending at a vertex. By another homotopy we may suppose $f$ is straight, i.e. that the path $f \mid e$ does not reverse direction in the middle of an edge. Thus $e$ is the union of a finite number of non-overlapping intervals that correspond homeomorphically to edges under $f$ unless $f \mid e$ is constant. If $e$ is oriented then we can form a formal product of oriented edges to describe the behaviour of $f$ on $e$. We justify this natural algebraic description of $f$ as follows.

We associate to $\Gamma$ its edge-path category EP whose objects are the vertices of $\Gamma$ and whose morphisms are certain concatenations of oriented edges of $\Gamma$. A morphism from $v_{1}$ to $v_{2}$ is an edge path from $v_{1}$ to $v_{2}$, i.e. a sequence of oriented edges $e_{1}, \ldots, e_{n}$, $n \geq 0$, where the back vertex of $e_{1}$ is $v_{1}$, the front vertex of $e_{i}$ is the back vertex of $e_{i+1}, i=0, \ldots, n-1$ and where the front vertex of $e_{n}$ is $v_{2}$. Taking $n=0$ gives the identity morphism at $v_{1}=v_{2}$. Composition is defined by concatenation:

$$
e_{1} \cdots e_{n} \circ e_{n+1} \cdots e_{m}=e_{1} \cdots e_{m}
$$

A straight $f: \Gamma \rightarrow \Gamma$ gives a map from the set of vertices of $\Gamma$ to itself and the image of an oriented edge $e$ determines a morphism $e_{1}, \ldots, e_{n}$ in the obvious way, where $n$ is the number of edges in the image of $f(e)$, counted with multiplicity. This extends to a product preserving map of EP that sends an identity morphism to another identity morphism, i.e. a functor from EP to itself. This functor which we denote $A f$ is the natural algebraic object underlying $f$. 
As an example, suppose $\Gamma$ has only one vertex $v$. Then any two morphisms can be composed. If we orient the edges $e_{1}, \ldots, e_{m}$ of $\Gamma$ and denote the oppositely oriented edges by $\bar{e}_{1}, \ldots, \bar{e}_{m}$ then EP is the free semigroup on the $e_{i}$ 's and $\bar{e}_{i}$ 's. To obtain $\pi_{1} \Gamma$ one adjoins the relations $e_{i} \bar{e}_{i}=\bar{e}_{i} e_{i}=i d_{i} ;$ to obtain $H_{1}(\Gamma ; \mathbb{Z})$ one adjoins further relations to say that $e_{i} e_{j}=e_{j} e_{i}$, all $i, j$. In each case $A f$ induces an algebraic operation that gives the induced map on $\pi_{1}$ or $H_{1}$. One can turn an arbitrary $\Gamma$ into such a graph by collapsing a maximal tree $T \subset \Gamma$ to a point. This gives the usual way to compute $\pi_{1} \Gamma$. Such collapsing, however, complicates the computation of the action of $f$ on twisted chains so we will proceed a little differently.

To each oriented edge $e$ we associate an indeterminate weight $x_{e}$. We let $H$ be the abelian group generated by the $x_{e}$ 's with the relations: ${ }^{\prime} x_{e_{1}} \cdots x_{e_{n}}=1$ whenever $e_{1}, \ldots, e_{n}$ is an edgepath from a vertex $v \in V$ to itself. Clearly $H$ is just a multiplicative copy of $H_{1}(\Gamma ; \mathbb{Z})$. There is a natural map $\pi: \mathrm{EP} \rightarrow H$ sending an oriented edge $e$ to $\pi(e)=x_{e}$. The functor $A f$ induces a map $f_{*}: H$ by the rule: if $A f(e)=e_{1} \cdots e_{m}$ then $f_{*}\left(x_{e}\right)=x_{e_{1}} \cdots x_{e_{m}}$. This $f_{*}$ is the usual induced map on $H_{1}(\Gamma ; \mathbb{Z})$. Let $R=\mathbb{Z} H$ be the integral group ring of $H$.

Recall that there is a covering space $\Gamma_{H}$ of $\Gamma$ with deck transformation group $H$ called the universal abelian cover of $H$. The map $f: \Gamma \rightleftharpoons$ lifts to $\Gamma_{H}$ (the commutator subgroup of $\pi_{1} \Gamma$ is characteristic) and one can easily construct an explicit representation for the algebraic part $A \tilde{f}$ of such a lift. This in turn determines the action of $\tilde{f}$ on the chain complex $C_{*}=C_{0}\left(\Gamma_{H}: \mathbb{Z}\right) \oplus C_{1}\left(\Gamma_{H} ; \mathbb{Z}\right)$ from which one can compute twisted zeta functions. It is not necessary explicitly to know $\Gamma_{H}$ (or even $H$ !).

Let us first illustrate this process. Let $\Gamma$ be a wedge of 3 circles with preferred orientations, $x, y$, and $z$. Then $\operatorname{EP}(\Gamma)$ is the free monoid with generators $x, \bar{x}, y, \bar{y}, z$ and $\bar{z}$. Let $f: \Gamma \rightleftharpoons$ be a map with

$$
\begin{aligned}
& A f(x)=y \bar{x}, \quad A f(\bar{x})=x \bar{y}, \\
& A f(y)=y z x y \bar{x}, \quad A f(\bar{y})=x \bar{y} \bar{x} \bar{z} \bar{y}, \\
& A f(z)=y, \quad A f(\bar{z})=\bar{y} .
\end{aligned}
$$

If is not hard to identify $H$ with $\left\{a^{i} b^{j} c^{k} \mid i, j, k \in \mathbb{Z}\right\}$ and $\Gamma_{H}$ with the 1-complex of all points in $\mathbb{R}^{3}$ with 2 or more coordinates in $\mathbb{Z}$. We choose a fundamental domain $D=\Gamma_{H} \cap[0,1)^{3}$. We denote the vertices and oriented edges in $D$ by $v_{H}, x_{H}, \bar{x}_{H}$, etc. and we denote the translation by $(i, j, k) \in \mathbb{Z}^{3}$ by $a^{i} b^{j} c^{k}=g$. The category $\operatorname{EP}\left(\Gamma_{H}\right)$ has as objects all $g v_{H}, g \in G$ and its morphisms are generated by $g x_{H}, g \bar{x}_{H}$, etc. We will lift $f$ to $f_{H}: \Gamma_{H} P$ so that $f_{H} v_{H}=v_{H}$ (this specifies the lift). One can check that $A f_{H}$ satisfies

$$
\begin{aligned}
& A f_{H}\left(v_{H}\right)=v_{H} ; \\
& A f_{H}\left(x_{H}\right)=y_{H} \cdot a^{-1} b \bar{x}_{H} ; \\
& A f_{H}\left(y_{H}\right)=y_{H} \cdot b z_{H} \cdot b c x_{H} \cdot a b c y_{H} \cdot b^{2} c \bar{x}_{H} ; \\
& A f_{H}\left(z_{H}\right)=y_{H} .
\end{aligned}
$$

In brief the pattern of $x$ 's, $\bar{x}$ 's etc. is as for $A f$ : only weights from $H$ have been inserted in a cumulative way. To check these weights, note that the rule 'front of one is back of the next' holds and that the three edgepaths all start at $A f_{H}\left(v_{H}\right)$, as 
they should. The free end of these edgepaths are, respectively, $a^{-1} b v_{H}, b^{2} c v_{H}$, and $b v_{H}$. This corresponds to the map $\phi=f_{*}$ on first homology $H$ induced by $f$

$$
\phi(a)=a^{-1} b, \quad \phi(b)=b^{2} c, \quad \phi(c)=b .
$$

To compute the action of $f_{H}$ on $C_{*}\left(\Gamma_{H}\right)$ it is enough to specify it on the preferred $R$-basis by

$$
\begin{aligned}
& f_{H^{*} v_{H}}=v_{H} \\
& f_{H^{*}} x_{H}=y_{H}-a^{-1} b x_{H} \\
& f_{H^{*}} y_{H}=y_{H}+b z_{H}+b c x_{H}+a b c y_{H}-b^{2} c x_{H} \\
& f_{H^{*} z_{H}}=y_{H}
\end{aligned}
$$

and to note the rule $f_{H^{*}}(g \cdot m)=\phi(g) \tilde{f}_{*}(m), m \in C_{*}\left(\Gamma_{H}\right)$.

Suppose one wishes to compute the action of $f$ on some other connected abelian cover $\tilde{\Gamma}$ of $\Gamma$. One has $\tilde{\Gamma}=\Gamma_{H} / S$ where $S$ is a subgroup of $H$. In order for $f$ to lift to $\tilde{\Gamma}$, one must have $\phi(S) \subset S$. Then the group $Q=H / S$ is the deck transformation group for $\tilde{\Gamma}$ over $\Gamma$ and $\phi$ induces a map $\psi: Q \rightarrow Q$ so that the lift $\tilde{f}=\left(f_{H}\right.$ modulo $\left.S\right)$. Formulae just like those above hold if one replaces the subscript $H$ by a superscript $\sim$ throughout and interprets $a, b, c$ as generators of $Q$ subject to the extra relations in $S$.

For example let $S$ be the subgroup generated by $b$ and $c$. Visibly it is $\phi$-invariant. $Q$ is infinite cyclic with generator $\eta$ corresponding to $a(\bmod S)$. We compute the action of $\tilde{f}: \tilde{\Gamma} \rightarrow \tilde{\Gamma}$ on chains from the $R^{\prime}$-basis $\tilde{v}, \tilde{x}, \tilde{y}, \tilde{z}$, where $R^{\prime}=\mathbb{Z} Q$, by the rule $\tilde{f}_{*}(q m)=q^{-1} \tilde{f}_{*}(m)$ and the table

$$
\begin{array}{ll}
\tilde{f}_{*}(\tilde{v})=\tilde{v}, & \tilde{f}_{*}(\tilde{x})=-\eta^{-1} \tilde{x}+\tilde{y}, \\
\tilde{f}_{*}(\tilde{y})=(1+\eta) \tilde{y}+\tilde{z}, & \tilde{f}_{*}(\tilde{z})=\tilde{y} .
\end{array}
$$

The cover corresponding to $Q$ is shown in figure 1 .

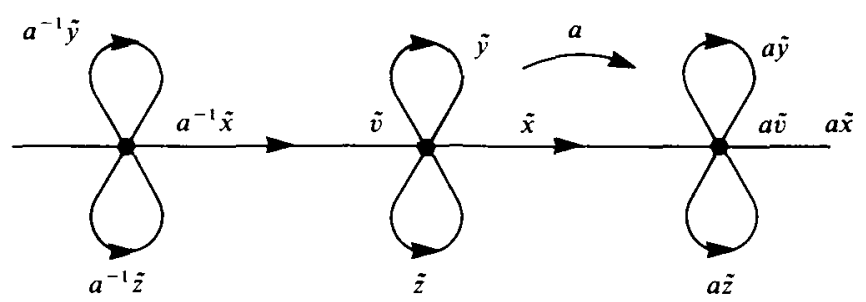

FIGURE 1

To continue the above computation, note that the square of $\psi$ is the identity on $Q$. This means that $\tilde{f}_{*}^{2}=F_{*}$ commutes with $Q$ and so defines a homomorphism of the $R^{\prime}$-module $C_{*}$. Relative to the preferred bases $\tilde{v}$ for $C_{0}$ and $\tilde{x}, \tilde{y}, \tilde{z}$ for $C_{1}$ we have

$$
F_{0}=(1), \quad F_{1}=\left(\begin{array}{ccc}
1 & 0 & 0 \\
1 & 3+\eta+\eta^{-1} & 1+\eta \\
1 & 1+\eta^{-1} & 1
\end{array}\right),
$$


as can be seen by iterating the above computation of $\tilde{f}$. Applying the definition of the twisted Lefschetz zeta function, one finds

$$
\tilde{\zeta}_{Q}\left(f^{2}, \xi\right)=1-\left(4+\eta+\eta^{-1}\right) \xi+\xi^{2} .
$$

Note that this is exactly the polynomial $p(\xi, \eta)$ of $\S 2$.

We now return to the general situation. To compute $A f_{H}$, one fixes a maximal tree $T$ (the vertex in the above example), a basepoint $p \in V \cap T$, and a preferred orientation for the edges of $\Gamma$. This gives a fundamental domain $D$ in $\Gamma_{H}$, determined (up to translation) by the property that $D$ contains a connected lift $\tilde{T}$ of $T$ and those edges of $\Gamma_{H}$ whose back vertex (in the preferred orientation lifted from $\Gamma$ ) lies in $\tilde{T}$. One chooses the lift $f_{H}$ to send the vertex $v_{H}$ in $\tilde{T}$ over $v$ into $\tilde{T}$. Then one inducts outward from $v$ in $T$ and inductively defines $A f_{H}$ : there is always a unique way to insert weights in $H$ into the terms in the formula for $A f$ so as to define an edge path in $\Gamma_{H}$ with the initial vertex given by earlier steps in the induction. This will be illustrated in $\S 6$.

To compute a zeta function it may be necessary to work in an intermediate abelian cover $\tilde{\Gamma}=\Gamma_{H} / S$ where $f$ lifts to $\tilde{\Gamma}$ and $\tilde{f}$ commutes with the deck transformation group $Q=H / S$. The above scheme works here just as well: $A \tilde{f}$ has weights determined uniquely by the same inductive process. Here $A \tilde{f}$ determines a chain map that is linear over $\mathbb{Z} Q$ and describable by finite matrices $F_{0}, F_{1}$ over $\mathbb{Z} Q$. $F_{0}$ has one non-zero entry in each column, and that is an element of $Q . F_{1}$ is obtained by abelianizing $A \tilde{f}$. Then $\tilde{\zeta}$ is computed, from the definition, as $\operatorname{det}\left(I-x F_{1}\right) / \operatorname{det}(I-$ $\left.x F_{0}\right)$.

\section{Blowing up}

We consider a pseudo-Anosov $h$ on an oriented connected compact surface $\Sigma$ such that $h$ preserves orientation. If $F \subset \Sigma$ is a finite invariant set, a new $\Sigma^{\prime}$ and $h^{\prime}$ with these same properties can be produced by blowing up the subset $F$. We compute the effect of this on the group $G(h)$ of $\S 2$.

Proposition 5. If $F$ contains $d$ orbits, $\Sigma$ has no boundary and $G(h)$ is finite then rank $G\left(h^{\prime}\right)=d-1$.

This means that by blowing up periodic orbits of $h$ we can increase the number of variables in the zeta function $\tilde{\zeta}(\varphi)$ and so broaden the collection of expansion constants produced in $\S 5$.

We will omit the elementary proof, but we will illustrate this proposition. First, let $\boldsymbol{A}$ be the toral automorphism induced by

$$
\left(\begin{array}{ll}
2 & 1 \\
1 & 0
\end{array}\right)
$$

We will take $\Sigma=T^{2}$,

$$
h=A^{2}=\left(\begin{array}{ll}
5 & 2 \\
2 & 1
\end{array}\right)
$$


$F=\operatorname{Fix}(A)=\left\{(0,0),\left(\frac{1}{2}, \frac{1}{2}\right)\right\}$ and $\Sigma^{\prime}, h^{\prime}$ as above. The proposition gives rank $G\left(h^{\prime}\right)=1$, since $\Sigma$ is closed, $F$ has 2 orbits and

$$
G(h)=\operatorname{coker}\left(\begin{array}{ll}
4 & 2 \\
2 & 0
\end{array}\right)
$$

is finite. By theorem 2 there is a twisted Lefschetz zeta function in two variables associated to $h^{\prime}$ that determines all the expansion constants in its flow equivalence class. Note that what we are doing is suspending the total automorphism $h$, blowing up a pair of closed orbits and forming new cross-sections, new return maps and new expansion constants.

To compute this zeta function, we compute the action of $h^{\prime}$ on $\pi_{1}\left(\Sigma^{\prime}, p\right)=\pi$. We choose the basepoint $p$ to be the point on $\partial \Sigma^{\prime}$ over $(0,0) \in \Sigma$ that corresponds to the eigendirection in the first quadrant. Then $\pi$ is freely generated by the paths $x, y, z$ of figure 2 . One computes $A_{*}^{\prime}: \pi \rightarrow \pi$ as

$$
\begin{aligned}
& A_{*}^{\prime}(x)=y x^{-1} ; \\
& A_{*}^{\prime}(y)=y z x y x^{-1} ; \\
& A_{*}^{\prime}(z)=y .
\end{aligned}
$$

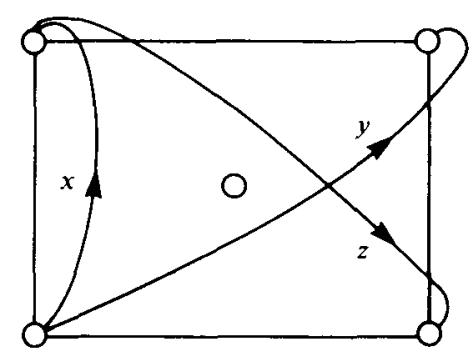

FIGURE 2

One can identify $\Sigma^{\prime}$ up to homotopy with a wedge of 3 circles and so pass from $h^{\prime}$ to the example $f$ of $\S 4$. Recalling the computation of $\tilde{\zeta}_{Q}\left(f^{2}, \xi\right)$ in that setting, we find

$$
\tilde{\zeta}_{G}\left(h^{\prime}, \xi\right)=1-\left(4+\eta+\eta^{-1}\right) \xi+\xi^{2}
$$

where $\eta$ is the class of $x$ in $G^{\prime}\left(h^{\prime}\right)=\left(H_{1}\left(\Sigma^{\prime} ; \mathbb{Z}\right) / y=z=0\right)=Q$. So the polynomial $p(\eta, \xi)$ is the zeta function of a pseudo-Anosov as promised in $\S 2$.

We remark that in the example above, the cover $\Sigma_{G}^{\prime}$ is not at all related to the cover of $\Sigma$ by $\mathbb{R}^{2}$. The maximal abelian cover $\Sigma_{H}^{\prime}$ has rank 3 and the map $\phi: H \rightarrow H$ induced by $h^{\prime}$ splits over $Q$ as a $q$-dimensional fixed subspace and a 2 -dimensional subspace that transforms by the matrix

$$
\left(\begin{array}{ll}
5 & 2 \\
2 & 1
\end{array}\right)
$$

Thus the obvious $\mathbb{Z}^{2}$ cover of $\Sigma^{\prime}$ is complementary to the $\mathbb{Z}$ cover we use. Whenever a hyperbolic toral automorphism is blown up on a set of fixed points and used in theorem 2, this will be the case. 
We observe that when $h: T^{2} \circlearrowright$ is a hyperbolic toral automorphism, any finite invariant set $F$ consists of points with rational coordinates [BR]. Thus, regarding $T^{2}$ as an additive group, there is an $n>0$ with $n \cdot F=0$. The subset $F_{n}$ of all points in $T^{2}$ of order dividing $n$ is a subgroup of $n^{2}$ elements that is invariant by $h$. For our purposes, one may always blow up more points (no expansion constants will be lost and some may be gained) so we can always work with an $F_{n}$ as $F$. We will take $n=2$ in $\S 6$.

This blowing up device has an application to the study of Anosov flows. If $\varphi$ is a transitive Anosov flow on a closed 3-manifold $M$ then a finite set of closed orbits can be blown up so that the new flow has a cross-section [F9]. It would be interesting to know if by blowing up enough orbits one can choose this cross-section to have genus 1 . This is known to be possible for the geodesic flow on a closed negatively curved surface [B], [F9]. As in the proposition, further blowing up can only increase the size of the flow equivalence class. The existence of a genus one section implies that $\varphi$ is a surgered version of a suspended toral automorphism.

\section{Expansion constants of odd degree}

We saw in $\S 4$ that there is a pseudo-Anosov $h^{\prime}: \Sigma^{\prime} \supseteq$ with $\tilde{\zeta}_{G}\left(h^{\prime}, \xi\right)=p(\eta, \xi)$. As noted in $\S 2$, the substitution $\eta=t^{a}, \xi=t^{b}$ with $|a|<b$ gives a Lefschetz zeta function for a pseudo-Anosov $r=r_{a b}: K_{a b} \rightleftharpoons$. If $a, b$ are relatively prime then $K_{a b}$ is connected.

We can rewrite $p(\eta, \xi)=(1-\xi)^{2}-(1+\eta)^{2} \eta^{-1} \xi$. If $a$ and $b$ have the same parity, then $\tilde{\xi}(r, t)$ is a difference of two squares and so will factor. We take $a=1$, $b=2 m+1, m \geq 1$. Then

$$
\tilde{\zeta}(r, t)=\left(t^{2 m+1}-t^{m+1}-t^{m}-1\right)\left(t^{2 m+1}+t^{m+1}+t^{m}-1\right) .
$$

The largest zero $\lambda>1$ of $\tilde{\zeta}(r, t)$ is a zero of the first factor, which we denote $p_{m}(t)$. This is obvious as the second factor exceeds $p_{m}(t)$ in this range.

It follows from [L] that $p_{m}(t)$ is irreducible. Thus we have exhibited an expansion constant of each odd degree. If $c, d$ are relatively prime positive integers of opposite parity, then the largest root of $t^{c+d}-t^{c}-t^{d}-1$ is also an expansion constant: choose $\xi=t^{c+d}, \eta=t^{c-d}$ and use [L] again to get irreducibility.

Let us examine more closely the degree 3 example corresponding to $K=K_{13}$ and $u \in H^{1}(M ; \mathbb{Z})$ given by $u(\eta)=1, u(\xi)=3$. We would like to know the topological type of $K$ and the configuration of singularities near the boundary. First we consider the boundary circle $\gamma$ in $\Sigma^{\prime}$ corresponding to $(0,0)$ in $\Sigma$. With the positive orientation for $\gamma$ and the basis $x, y, z$ for $\pi_{1}\left(\Sigma^{\prime}, p\right)$ as in the previous section, we find that $\gamma$ represents $x y^{-1} z x$, and so corresponds to $\eta^{2} \in H$. Thus the restriction $i^{*} u$ of $u$ to the toral boundary component $T(\gamma)$ swept out by $\gamma$ assigns 2 to the meridian curve $\gamma$ and 3 to the longitude curve represented by the closed orbit $\delta$ through $p$. Hence $i^{*} u$ is irreducible and $K_{13}$ meets $T(\gamma)$ in a connected 1-manifold, i.e. a circle. Moreover the boundary component $K_{13} \cap T(\gamma)$ meets $\delta 3$ times. So, recalling that $\delta$ is one of 4 parallel closed orbits on $T(\gamma)$ corresponding to the 4 fixed directions at $(0,0) \in \Sigma$, we find that $K_{13} \cap T(\gamma)$ is as shown in figure 3. The map $r$ cyclically 

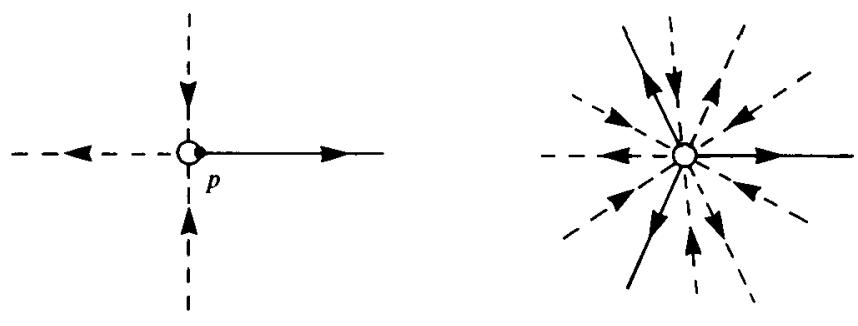

FIGURE 3

permutes the three heavily shaded prongs that correspond to the stable manifold of $\delta$.

We will see that the same thing happens at the other boundary component of $M_{h^{\prime}}$. This is the torus $T\left(\gamma^{\prime}\right)$ swept out by the circle $\gamma^{\prime}$ corresponding to $\left(\frac{1}{2}, \frac{1}{2}\right) \in \Sigma$. The homology class of $\gamma^{\prime}$ is the opposite of that of $\gamma$ in $H_{1}\left(\Sigma^{\prime} ; \mathbb{Z}\right)$ since $\gamma+\gamma^{\prime}$ bounds. This implies that $T\left(\gamma^{\prime}\right) \cap K_{13}$ is connected, imitating the preceding argument. If $\delta^{\prime}$ is the orbit through a fixed point $p^{\prime} \in \gamma^{\prime}$, we would like to know the homology class $\xi^{k} \eta^{l}$ of $\delta^{\prime}$. Clearly $k=1$ since $\delta^{\prime}$ cuts $\Sigma^{\prime}$ once. To find $l$, one draws a path $\varepsilon$ from $p$ to $p^{\prime}$ and counts the number of occurrences of $x$ in the loop $\varepsilon f(\varepsilon)^{-1}$. One finds (figure 4)

$$
\varepsilon f(\varepsilon)^{-1}=x y^{-1} x^{-1} z^{-1} y^{-1}
$$

so $l=0$. Thus $\delta^{\prime}$ has the same homology class $\xi$ as $\delta$, and the same computation applies.

We also know that the degree of the zeta function of $r$ is the negative of the Euler characteristic of $K$. So $K$ has $\chi=-6$, two boundary components, and is orientable; it is a genus 2 surface with two holes. Collapsing these boundary circles gives a closed genus 2 surface $S$ and a pseudo Anosov $f: S \rightarrow S$ with 2 singular points, each with 6 stable prongs, each prong of period 3 under $f$. The expansion constant $\lambda$ for $f$ satisfies $\lambda^{3}=\lambda^{2}+\lambda+1$.

Such an example (same genus, same $\lambda$, same singular behaviour) was also given by Arnoux and Yoccoz by a completely different construction. It is not known

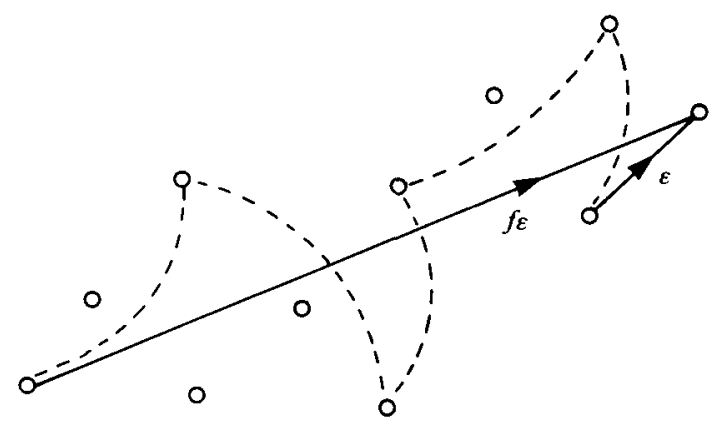

Figure 4 
whether these two examples are the same, but it seems very probable that they are. They produced $\lambda$ 's satisfying $\lambda^{d}=\lambda^{d-1}+\cdots+\lambda+1$ for any $d \geq 3$, so only their $d=3$ case agrees with our $\lambda$ 's $[\mathbf{A Y}]$.

Our examples have a different root distribution from theirs. Their $\lambda$ 's are Pisot, i.e. all their non-trivial algebraic conjugates lie in the unit disc. But

Proposition 6. The roots of $p_{m}(t)$ are divided almost equally by the unit circle: $m$ are on one side, $m+1$ on the other.

Proof. Fix $m$. Consider the family $q_{c}(t)=t^{2 m+1}-c\left(t^{m+1}+t^{m}\right)-1, c \in \mathbb{R}$. One sees that $q_{c}\left(t^{-1}\right) t^{2 m+1}=q_{-c}(t)$, so the reciprocals of the roots of $q_{c}$ are roots of $q_{-c}$ Since $t^{2 m+1}-1$ and $t^{m+1}+t^{m}$ are relatively prime, $q_{c}(t)$ is relatively prime to $q_{-c}(t)$ for $c \neq 0$. Thus for $c \neq 0$ no zero $z$ of $q_{c}(t)$ can be on the unit circle: if $|z|=1$ then $z^{-1}$ is a root of $q_{-c}$ (by the above) and of $q_{c}$ (as $q_{c}$ is real and $z^{-1}=\bar{z}$ ), which would give a common factor $t-z^{-1}$ for $q_{-c}$ and $q_{c}$

As the roots of $q_{c}(t)$ vary continuously with $c$, and as $p_{m}(t)=q_{1}(t)$, it suffices to see how the roots of $q_{0}(t)=t^{2 m+1}-1$ move off the unit circle at $c=0$.

Fix $k \in\{0,1, \ldots, 2 m\}$ and let $z(c)$ be the solution of $q_{c}(t)$ that equals $\rho=$ $\exp 2 \pi i k /(2 m+1)$ at $c=0$. As $q_{0}(t)$ has distinct roots, $z(c)$ is differentiable for small $c$. Differentiating the identity $q_{c}(z(c))=0$ with respect to $c$ at $c=0$ gives

$$
(2 m+1) \rho^{2 m} z^{\prime}=\rho^{m+1}+\rho^{m} .
$$

We want to know whether the modulus of $z(c)$ is increasing or decreasing at $c=0$, i.e. whether $z^{\prime} / \rho$ has positive or negative real part. We have shown that

$$
\operatorname{Re}\left(z^{\prime} / \rho\right)=\frac{2}{2 m+1} \operatorname{Re}\left(\rho^{m}\right)
$$

Letting $k$ vary we see that $\rho^{m}$ sweeps out all the $(2 m+1)$ st roots of unity. Thus as many roots move out of the unit circle as there are $(2 m+1)$ st roots of unity with positive real part.

\section{A larger example}

We will develop here an example of theorem 2 on a 3-manifold with first Betti number $\beta=4$. We will begin by some general considerations concerning the use of punctured toral automorphisms in flow equivalence.

We will take a hyperbolic toral automorphism $\alpha: T^{2} \rightarrow T^{2}$, blow it up on a finite invariant set $E$, suspend and take a new cross-section. We will ensure that $\alpha$ preserves the stable and unstable orientations, i.e. both its eigenvalues will be positive. One eigenvalue is $\lambda(\alpha)$, the other is $\lambda(\alpha)^{-1}$. We may simplify $\alpha$ somewhat by choosing a good basis for the lattice $\mathbb{Z}^{2}$ whose quotient gives us $T^{2}$. For a suitable choice of integral basis for $\mathbb{Z}^{2}$, one may factor $\alpha$ as a (positive) word in the transvections

$$
\sigma=\left(\begin{array}{ll}
1 & 1 \\
0 & 1
\end{array}\right), \quad \tau=\left(\begin{array}{ll}
1 & 0 \\
1 & 1
\end{array}\right)
$$

[MKS]. Next one may simplify $E$ as in $\S 4$, by replacing it by a larger invariant set $E_{n}=\left\{x \in T^{2} \mid n x=0\right\}$ for appropriate $n \geq 1$. Let $\Sigma_{n}$ be the surface obtained by blowing 
up $T^{2}$ at $F_{n}$, so $\Sigma_{n}$ has genus 0 and $n^{2}$ boundary components. The blown up version of $\alpha$, call it $\alpha_{n}$, can be viewed as a product of blown up transvections $\sigma_{n}, \tau_{n}$. This simplifies the computation of the action of $\alpha_{n}$ on the universal abelian cover of $\Sigma_{n}$. Also one can choose a cell complex $\Gamma_{n}$ of dimension 1 with the same homotopy type as $\Sigma_{n}$ as follows. Consider the grid $\Gamma_{\infty}=\left\{(x, y) \in \mathbb{R}^{2} \mid x \in \mathbb{Z}\right.$ or $\left.y \in \mathbb{Z}\right\}$. It is a sort of infinite piece of graph paper with a natural $\mathbb{Z}^{2}$ action. Let $\Gamma_{n}=\Gamma_{\infty} / n \cdot \mathbb{Z}^{2}$ be the quotient by the action of the subgroup of elements divisible by $n$. We can label the vertices of $\Gamma_{n}$ as $P_{i j}$, and the edges as $h_{i j}, v_{i j}$ and where $i, j$ vary over $\mathbb{Z} / n \mathbb{Z}$. Here $P_{i j}$ is the point $(i, j)\left(\bmod n \mathbb{Z}^{2}\right), v_{i j}$ is the vertical edge $\{(i, y) \mid j \leq y \leq j+1\}$ and $h_{i j}$ is the horizontal edge $\{(x, j) \mid i \leq x \leq i+1\}$. We identify $\Gamma_{n}$ with a subcomplex of $\Sigma_{n}$ as in figure 5 . We can then rectify $\sigma_{n}$ by the rule

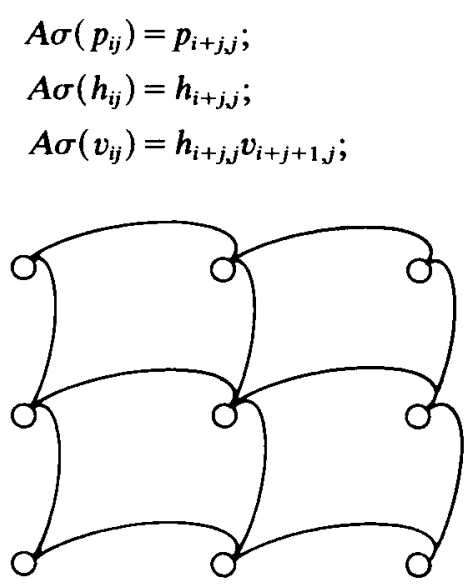

Figure 5

or, more concisely, ' $h \rightarrow h, v \rightarrow h v$ '. Similarly one can define the functor $A \tau$ by the symmetrically related scheme $A \tau\left(p_{i j}\right)=p_{i, i+j}$ and ' $h \rightarrow v h, v \rightarrow v$ '. With this any $\alpha_{n}$ can be computed using only positive words in $h$ and $v$. The method of $\S 3$ gives an algorithm for computing the action of $\alpha_{n}$ on the maximal abelian cover of $\Sigma_{n}$ (note this cover has deck group $H_{n}$ of rank $n^{2}+1$ ), once a maximal tree $T$ and basepoint $p$ are chosen in $\Sigma_{n}$. This basepoint $p_{\infty}$ stands out but there is no natural choice of $T$. One may use, say, the comb

$$
T=(0 \times[0, n-1]) \cup([0, n-1] \times\{0,1, \ldots, n-1\}) .
$$

We now specialize to $n=2$ and

$$
\alpha=\left(\begin{array}{ll}
5 & 2 \\
2 & 1
\end{array}\right)=\sigma^{2} \tau^{2}
$$

Since

$$
\alpha \equiv\left(\begin{array}{ll}
1 & 0 \\
0 & 1
\end{array}\right)(\bmod 2),
$$

$\alpha$ fixes $E_{2}$. As in $\S 4$, this implies that $G\left(\alpha_{2}\right)$ has rank 3. We choose a fundamental domain as in $\$ 3$ and label its edges as shown in figure 6. The small letters denote 


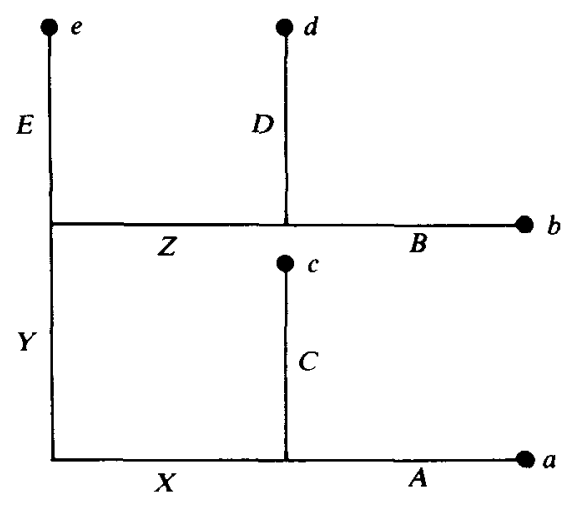

Figure 6

the weights $a, b, c, d, e \in \mathrm{H}_{2}$ corresponding to $A, B, C, D, E$ : no weights are needed on the tree $X, Y, Z$ since $H_{2}$ is rank 5. Note that $\sigma^{2}, \tau^{2}$ also fix $E_{2}$, so their representations on $\Gamma_{2}$ fix all 4 vertices. We compute the edgepath functors induced by $\sigma^{2}$ and $\tau^{2}$ as in table 1 . To construct table 1 , the edges were ordered outward from the origin, starting with those in $T$. Then all the capital letters in the other columns were filled in using the $v, h$ symbolic descriptions. For example if one applies $\sigma^{2}$ to $Y$ then its back vertex is fixed and since $Y$ is vertical it will go to ' $h{ }^{2} v$ '. Starting at the back vertex of $Y$, one goes right 2 steps and up one, tracing through $X, A, Y$ in that order. Finally the weights were filled in: it is here that ordering the edges was important. For instance, the term $Y$ in $\sigma_{2}^{2}(Y)$ occurs after an $A$, so a weight $a$ is put in. The weights accumulate. Since $E$ begins at the end of $Y$, the $Z$ in $\sigma_{2}^{2}(E)$ begins with the weight $a$ that $Y$ gave it. Implicit in this table is another one showing what weights the various vertices pick up. We have not included it, in order to be concise, and it can easily be deduced from the table. For instance since the image of $E$ carries a weight $a$ in the first term, the back vertex of $E$ goes to itself with the weight $a$.

From table 1 for the chain map $F$, one could calculate $\operatorname{det}\left(I-x F_{1}\right)$ and $\operatorname{det}(I-$ $x F_{0}$ ) by brute force and so compute $\tilde{\zeta}_{G}$ It is better to proceed as follows.

TABLE 1

\begin{tabular}{lll}
\hline edge & $\sigma_{2}^{2}: v \rightarrow h^{2} v, h \rightarrow h$ & $\tau_{2}^{2}: v \rightarrow v, h \rightarrow v^{2} h$ \\
\hline$X$ & $X$ & $Y+E+e X$ \\
$Y$ & $X+A+a Y$ & $Y$ \\
$Z$ & $a Z$ & $E+e Y+e Z$ \\
$A$ & $A$ & $e C+e c D+e c d A$ \\
$B$ & $a B$ & $e D+e d C+e d c B$ \\
$C$ & $A+a X+a C$ & $e C$ \\
$D$ & $a B+a b Z+a b D$ & $e D$ \\
$E$ & $a Z+a B+a b E$ & $E$
\end{tabular}


First we compute $G$. In order that the weights be conserved by $\sigma_{2}^{2}$ one must have $a b=1$. For instance the cycle $Y E$ goes to $X A Y Z B E$. Equating the corresponding products of weights gives $e=a b e, a b=1$. This is the only relation imposed by $\sigma_{2}^{2}$ but $\tau_{2}^{2}$ gives the relation $c d e=1$. This gives a rank 3 quotient group $G$ on which $\sigma_{2}^{2} \tau_{2}^{2}$ acts trivially. As we know there is a complementary rank 2 group where the action is hyperbolic, this $G$ is the largest such quotient group. Henceforth we will regard the weights as in $G=\langle a, b, c, d, e| a b=c d e=1$, all generators commute $\rangle$.

Second, the matrix $F_{0}$ is diagonal, since $\sigma^{2}$ and $\tau^{2}$ fix all vertices. From table 1 one sees that the back vertex of $E$, for example, scales successively by $a$ and 1 , so $a$ is an eigenvalue of $F_{0}$. Altogether one has diagonal entries $1, a, e, a e$.

Recall that there is a boundary operator $\partial$ over $R=\mathbb{Z} G$ such that $\partial F_{1}=F_{0} \partial$. Up to torsion for the integral domain $R, \partial$ is surjective. For example, $\partial(Y+E)=$ $(e-1) v_{\infty}$. Consider the quotient field $L$ of $R$. Viewing $R$ as $\mathbb{Z}\left[a, a^{-1}, c, c^{-1}, e, e^{-1}\right]$, $L$ is the rational function field $Q(a, c, e)$. The above relation shows $(e-1) v_{\infty}$ bounds and so, since $e-1$ is invertible in $L, v_{\infty}$ bounds too. Altogether, one sees that $1, a, e$ and $a e$ are also eigenvalues of $F_{1}$. So $\tilde{\zeta}_{G}$ is a polynomial of degree four over $R$.

We will need to compute some coefficients of $\operatorname{det}\left(I-x F_{1}\right)=$ $1-T_{1} x+T_{2} x^{2}-\cdots+T_{8} x^{8}$ where $T_{i}=$ Trace $\Lambda^{i}\left(F_{1}\right)$. Note that $\Lambda^{i} F_{1}=\Lambda^{i} M \cdot \Lambda^{i} N$ where $M$ is the matrix that represents $\tau^{2}$ and $N$ that which represents $\sigma^{2}$, each given by the appropriate column in our table. Recalling the usual inner product on matrices is $\langle\alpha, \beta\rangle=$ Trace $\alpha \beta^{t}$, we have $T_{i}=\left\langle\Lambda^{i} M,\left(\Lambda^{i} N\right)^{t}\right\rangle$, a simpler expression to compute since one need not multiply large matrices. We display $M$ and $N^{\prime}$, relative to the convenient ordering of our basis as $X, A, B, Z: Y, C, D, E$ in figure 7 . It is clear that $M, N^{t}$ have determinants $e^{4}, a^{4}$ so $T_{8}=e^{4} a^{4}$. Also $T_{1}=\left\langle M, N^{t}\right\rangle$ contains a contribution $\delta=(e+1+a+a e+a+a e+e+1)$ from the diagonal terms and $\nu=$ $(1+e+a e+a)=\delta / 2$ from the terms in the $4 \times 4$ block lying on its diagonal.

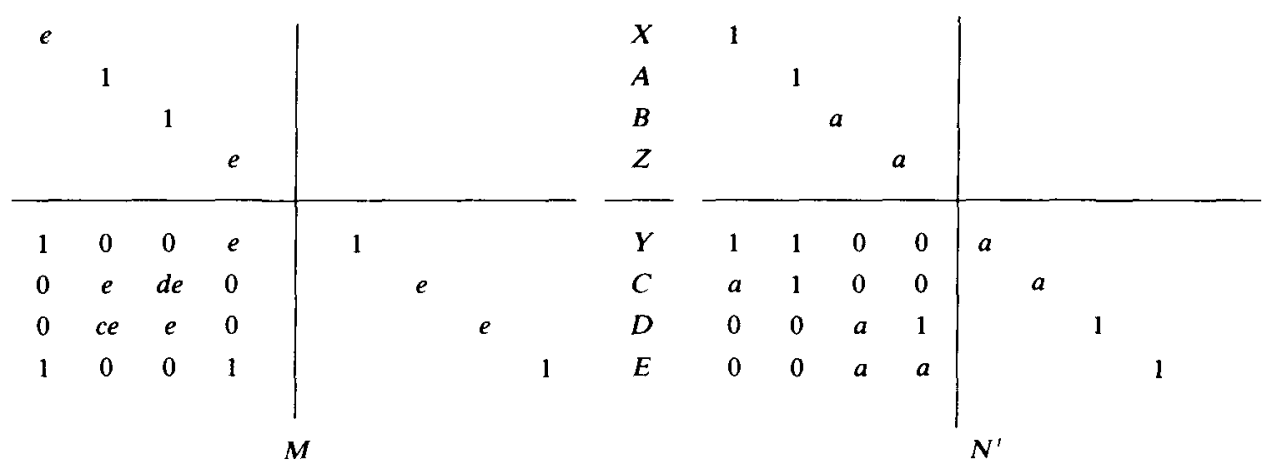

FIGURE 7

It will be shown in $\S 7$ that $\tilde{\zeta}_{G}$ is reciprocal, i.e. under the ring automorphism sending $a \rightarrow a^{-1}, c \rightarrow c^{-1}, e \rightarrow e^{-1}, x \rightarrow x^{-1}, \tilde{\zeta}_{G}$ maps to a multiple of itself by a trivial unit of the form $\pm g x^{k}, g \in G, k \in \mathbb{Z}$. With these elementary facts we can almost compute $\tilde{\zeta}_{G}$. We have

(*) $1-T_{1} x+T_{2} x^{2}-\cdots=(1-x)(1-x a)(1-x e)(1-x a e) \tilde{\zeta}_{G}$ 
and we know $T_{1}, T_{8}$. Computing $(*)$ modulo $x^{2}$ and writing $\tilde{\zeta}_{G}=$ $1-k_{1} x+k_{2} x-k_{3} x+k_{4} x^{4}$ we find $T_{1}=k_{1}+\nu$, so $k_{1}=\delta$. Also $(*)$ implies $e^{4} a^{4}=$ $(a e)^{2} k^{4}$, so $k_{4}=e^{2} a^{2}$. As $k_{3}$ and $k_{1}$ must be symmetric around $\sqrt{k_{4}}=e a$, we find $k_{3}=e a \delta$. Only $k_{2}$ is not known, but it can be computed from $T_{2}$.

At first this seems difficult but the matrices $M, N^{t}$ are so sparse that it is not. One need not, of course, write out $\Lambda^{2} M$ and $\Lambda^{2} N^{t}$ to take their inner product. One considers $2 \times 2$ blocks and groups them according to the number of diagonal entries that occur, 0,1 or 2 , so $T_{2}=S_{0}+S_{1}+S_{2}$. Those with zero diagonal entries come from the $4 \times 4$ corner block: one finds its 6 principal minors and only 2 non-principal ones make a contribution

$$
\begin{aligned}
S_{0}= & {\left[e(1-a)+e a+(1-e) a+\left(e^{2}-c d e^{2}\right) a+e a+e\left(a^{2}-a\right)\right] } \\
& +\left[-c e^{2} \cdot 1+-c d e \cdot a^{2}\right] .
\end{aligned}
$$

The contributing terms with 1 diagonal entry must have their opposite entry on the diagonal of the corner block, thanks to the distribution of the zero entries in $M$ and $N^{t}$. Taking the diagonal entries in order gives

$$
\begin{aligned}
S_{1}= & e(e+e a+a)+1(1+e a+a)+a(1+e+a)+a e(1+e+e a) \\
& +a(e+e a+a)+a e(1+e a+a)+e(1+e+a)+1(1+e+e a) \\
= & \nu^{2}+(a+e)^{2}+(1+a e)^{2} .
\end{aligned}
$$

One computes $S_{2}$ as $\frac{1}{2}$ the crossterms in $\delta^{2}$. Thus

$$
S_{2}=2(1+a)^{2}(1+e)^{2}-\left(1+a^{2}\right)\left(1+e^{2}\right) .
$$

We now compute $k_{2}$ from the quadratic terms in $(*)$. After simplification one finds

$$
\tilde{\zeta}_{G}=1-2 \nu x+\left(\nu^{2}-c e^{2}-c^{-1} a^{2}\right) x^{2}-2 a e \nu x^{3}+a^{2} e^{2} x^{4} .
$$

Note that, as anticipated, the middle term is symmetric about ae.

We now draw some consequences from this formula. First $\tilde{\zeta}_{G}$ is almost a difference of squares:

$$
\tilde{\zeta}_{G}=\left(1-\nu x+a e x^{2}\right)^{2}-x^{2}(a+e c)^{2} c^{-1} .
$$

So we substitute $\eta^{2}$ for $c$ and find the factors

$$
1-\nu x+a e x^{2} \mp x\left(a+e \eta^{2}\right) \eta^{-1}=(1-a x)(1-e x)-x(1 \pm a \eta)\left(1 \pm e \eta^{-1}\right) .
$$

We see from proposition 4 that the cone $\mathscr{C}_{\varphi}$ is defined by $u\left(\operatorname{Supp}^{\prime} \tilde{\zeta}_{G}\right)>0$. So, in this example, $\mathscr{C}_{\varphi}$ is defined by $\left\{a x, e x, a e x^{2}, x, a e x, a \eta x, e \eta^{-1} x\right\}$. Of these 7 points only $a e x^{2}$ is expressible as a non-negative combination of the others, so $\mathscr{C}$ has 6 distinct faces. On the other hand $\varphi$, the suspension flow of $\alpha_{2}$,

$$
\alpha=\left(\begin{array}{ll}
5 & 2 \\
2 & 1
\end{array}\right),
$$

has only 4 homology classes of singular orbits, corresponding to the 4 boundary components. So the homology classes of singular orbits do not define $\mathscr{C}_{\varphi}$. In particular they do not determine the existence of cross-sections nor a face of the unit ball in Thurston's norm on $H^{1}$ [F2], [T2]. This answers a question posed in 1977 by members of the Orsay topology seminar. 
If we take the proper sign in the above factoring and let $a=e=1, x=\xi$, we get the polynomial $p(\xi, \eta)$ studied above. This is because setting $a=e=1$ corresponds to collapsing the fixed points $\left(0, \frac{1}{2}\right)$ and $\left(\frac{1}{2}, 0\right)$, returning us to the example of $\S 5$.

Other substitutions give quite different $\lambda$ 's. For instance, let $\eta=a=1, x=t, e=t^{2}$. Then $\tilde{\zeta}=(1-t)\left(1-t^{3}\right) q(t)$, where $q(t)=1-3 t-3 t^{3}+t^{4}$. Clearly $\lambda$ is a zero of $q(t)$. Substituting $v=t+t^{-1}$, one finds $t^{-2} q(t)=Q(v)$ where $Q(v)=v^{2}-3 v-2$. Now $Q$ is irreducible and has a zero in $(-2,2)$. Thus $q$ has a zero on the unit circle (see [F8]). It is not hard to check that $q$ is irreducible, so that the expansion constant $\lambda$ has an algebraic conjugate on the unit circle. Considering the hyperbolic behaviour of pseudo-Anosovs, this is surprising behaviour.

The method of computation applied above to $\alpha^{2}$ would work just as well for $\left(\sigma_{2}^{2}\right)^{m}\left(\tau_{2}^{2}\right)^{n}, m, n>0$. For $\left(\sigma_{2}^{2}\right)^{m},\left(\tau_{2}^{2}\right)^{n}$ have the same distribution of zeros that led to a simple computation of the determinant. One could write a closed form expression for the zeta functions of $\left(\sigma^{2 m} \tau^{2 n}\right)_{2}$, all $m, n>0$. For examples more complicated than this it might be wise to use a computer language like MACSYMA that does symbolic manipulations. The group generated by $\sigma_{2}^{2}, \tau_{2}^{2}$ is identified in [S].

\section{Some remarks on flow equivalence}

We return to the general situation of a compact 3-manifold $M$ with a circular flow $\varphi$. We will prove a symmetry property for $\tilde{\zeta}_{H}(\varphi)$ and study analytically how the growth rates of cross-sections vary over $\mathscr{C}_{\varphi}$. Finally we describe flow equivalence in a way that makes no direct use of flows.

The symmetry property is:

Proposition 7. For $\varphi$ as above and $M$ oriented, the involution of $\mathbb{Z} H$ that sends $h \rightarrow h^{-1}$ carries $\tilde{\zeta}_{H}(\varphi)$ to $\pm h_{0} \cdot \tilde{\zeta}_{H}(\varphi)$, for some $h_{0} \in H$.

Proof. Choose an integral basis $x_{1}, \ldots, x_{\beta}$ for $H$. Then consider the homeomorphisms $u: H \rightarrow \mathbb{Z}$ and the rational functions $R_{u}$ in one variable $t$ obtained by substituting $t^{u\left(x_{i}\right)}$ for $x_{i}, i=1, \ldots, \beta$. If $\tilde{\zeta}_{H}(\varphi)$ were not reciprocal in the above sense then those $u$ 's such that $\boldsymbol{R}_{\boldsymbol{u}}$ is reciprocal would lie in finitely many proper rational subspaces of $\operatorname{Hom}(H, \mathbb{Q})=H^{1}(M ; \mathbb{Q})$. Now on the open cone $\mathscr{C}_{\varphi}, R_{u}$ is just the Lefschetz zeta function of the return map $r$ for a cross-section dual to $u$. To conclude the argument, we observe that for any orientation preserving surface homeomorphism $r, \tilde{\zeta}(r, t)$ is reciprocal. For closed surfaces this is immediate from Poincare duality [Fr]. If there are boundary components then collapsing them only changes $\tilde{\zeta}$ by reciprocal factors $1-t^{n}$ (cf. [F10]).

Another proof of this proposition could be based on the Reidemeister torsion of the abelian covering $M_{H}[\mathbf{F 4}],[\mathbf{M}]$. We prefer the more dynamic argument in this situation.

Without using zeta functions, one can see that $\lambda$ varies under flow equivalence. We have:

Proposition 8. Let $\varphi$ be a circular pseudo-Anosov flow. The function $(\log \lambda)^{-1}$ : $\mathscr{C}_{\varphi} \cap H^{1}(M ; \mathbb{Z}) \rightarrow(0, \infty)$ extends to a concave, homogeneous function $\mathscr{C}_{\varphi} \rightarrow[0, \infty)$ that vanishes on $\partial \mathscr{C}_{\varphi}$. 
Proof. For any pseudo-Anosov $r$, the topological entropy $h(r)$ is $\log \lambda$, where $\lambda$ is the expansion constant [FS]. The function $h(r)^{-1}$ was shown to have the desired properties in [F3].

This concavity is not obvious from the zeta function description of growth rates, as it depends on the description of $\lambda$ using a Markov partition. It is easy to see that the map $l(u)=-\chi\left(K_{u}\right)$ is linear in $u$ and positive on $\mathscr{C}_{\varphi}$ [F2]. It was shown in [F2] that $l(u)$ is positive on the closure $\overrightarrow{\mathscr{C}}_{\varphi}$. So we see that the function $(\log \lambda)^{-1} / l(u)$ is bounded above.

It would be interesting to know whether the concavity property of $\lambda$ holds for higher dimensions.

Problem 3. Let $\varphi$ be a circular flow on a compact manifold $M$. Is the function $(\log \lambda)^{-1}: \mathscr{C}_{\varphi} \cap H^{1}(M ; \mathbb{Z}) \rightarrow(0, \infty]$ concave?

This is a purely group theoretic problem but should probably be dealt with geometrically.

We conclude with a description of flow equivalence that doesn't involve 3manifolds. Take $f: \Sigma \rightarrow \Sigma$ a surface homeomorphism and $\Lambda \subset M_{f}$ a cross-section to the suspension flow $\varphi$ of $f$. The class $u_{\Lambda} \in H^{1}\left(M_{f} ; \mathbb{Z}\right)$ dual to $\Lambda$ restricts to a class $\xi \in H^{1}(\Sigma ; \mathbb{Z})$ that determines a $\mathbb{Z}$-cover $\hat{\Sigma}$ of $\Sigma$. The Wang sequence for $\theta$ shows $f^{*} \xi=\xi$. Thus $f$ lifts to $\hat{\Sigma}$ and the lifts commute with deck transformations. Let $\hat{f}$ be such a lift and $g$ the preferred generator for the deck transformation group. We will show there is a basis $(m, n),(a, b)$ for $\mathbb{Z}^{2}$ such that the elements $\gamma=\hat{f}^{m} g^{n}$, $\hat{h}=\hat{f}^{a} g^{b}$ saiisfy:

(i) the group $H_{\gamma}$ generated by $\gamma$ acts properly discontinuously on $\hat{\Sigma}$ with $\Lambda$ as quotient;

(ii) the map of $\Lambda$ induced by $\hat{h}$ is $h$.

Thus two flow equivalent transformations are obtained from a 2-ended surface $\hat{\Sigma}$ with a $\mathbb{Z}^{2}$ action by factoring by properly discontinuous $\mathbb{Z}$ subactions in two distinct ways.

Rather than exhibiting $(m, n)$ and $(a, b)$ it is more instructive to construct $\hat{\Sigma}$ directly from the geometry. Let $M^{*}$ be the $\mathbb{Z}^{2}$-cover of $M_{f}$ corresponding to the pair $\left(u_{\Sigma}, u_{\Lambda}\right)$. Let $\varphi^{*}$ be the flow on $M^{*}$ which covers $\varphi$. The $\mathbb{Z}$-cover $\Sigma \times \mathbb{R}$ of $M_{f}$ is obtained by factoring $M^{*}$ by one $\mathbb{Z}$ factor. Symmetrically, $\Lambda \times \mathbb{R}$ is also an intermediate $\mathbb{Z}$-cover. The flow $\varphi^{*}$ corresponds (up to reparametrization) with the standard flows on $\Sigma \times \mathbb{R}, \Lambda \times \mathbb{R}$. So the orbit space $\mathcal{O}$ of $\varphi^{*}$ is a $\mathbb{Z}$-cover of $\Sigma$ and a $\mathbb{Z}$-cover of $\Lambda$. Thus $\mathcal{O}$ is the space $\hat{\Sigma}$ of the preceding paragraph. The $\mathbb{Z}^{2}$ action on $\sigma$ is induced from the deck transformations of $M^{*}$ (which commute with $\varphi^{*}$ since $\varphi^{*}$ was constructed as a lifted flow).

One can describe the entire flow equivalence class of $f: \Sigma \rightleftharpoons$ in this way. Let $G=G(f)$ as in $\S 2$ and form the corresponding cover $\Sigma_{G}$. Then $f$ lifts to $f_{G}: \Sigma_{G} \rightleftharpoons$ and the lift $f_{G}$ commutes with the deck transformation group $G$. Combining these lifts and the deck transformations gives the abelian group $H$ of all lifts of all iterates of $f$ ( $G$ is exactly the largest torsionfree quotient of $H_{1}(\Sigma ; \mathbb{Z})$ such that this $H$ is abelian). So one has a $\mathbb{Z}^{\beta}$ action with a properly discontinuous cocompact subaction of $\mathbb{Z}^{\beta-1}$. For certain other splittings of $H$ as a sum of a rank $\beta-1$ group $G^{\prime}$ and a 
rank 1 group $\left\{h^{i} \mid i \in \mathbb{Z}\right\}, G^{\prime}$ acts properly discontinuously on $\Sigma_{G}$. Then the compact surface $\Sigma^{\prime}=\Sigma_{G} / G^{\prime}$ carries a homeomorphism $f^{\prime}$ induced by $h$. If the generator $h$ was chosen properly, $f^{\prime}$ is flow equivalent to $f$ and all flow equivalent homeomorphisms arise from such splittings of $H$. This description follows from studying the orbit space of the lifted flow on the cover $M_{H}$, i.e. the maximal free abelian cover of the mapping torus $M=M_{f}$.

This suggests a natural way to extend the notion of flow equivalence to actions of groups other than $\mathbb{Z}$. Let $H$ be a group that acts on a manifold $X$. Let $G_{i} \triangleleft H$, $i=1,2$, be normal subgroups of $H$ such that the action of $G_{i}$ on $X$ is free and properly discontinuous for $i=1,2$. Then the manifolds $Y_{i}=X / G_{i}$ carry induced actions of $\Gamma_{i}=H / G_{i}$. We say that the action of $\Gamma_{1}$ on $Y_{1}$ is cover equivalent to the action of $\Gamma_{2}$ on $Y_{2}$. In the case where $\Gamma_{1}$ and $\Gamma_{2}$ are infinite cyclic, appropriate generators of $\Gamma_{1}, \Gamma_{2}$ are flow equivalent. In general, we do not necessarily have $\Gamma_{1}$ isomorphic to $\Gamma_{2}$, even if both $Y_{i}$ are compact. Cover equivalent actions should be interesting objects of study as topological analogues of the orbit equivalent actions in ergodic theory.

This research was partially supported by the National Science Foundation and Sloan Foundation.

\section{REFERENCES}

[AY] P. Arnoux \& J. C. Yoccoz. Construction de diffeomorphisme pseudo-Anosov. C.R. Acad. Sci. Paris 292 (1981), 75-78.

[BR] T. Banchoff \& M. Rosen. Periodic points of Anosov diffeomorphisms. In Global Analysis XIV, Proc. Symp. Pure Math. Amer. Math. Soc.: Providence, 1970.

[B] G. D. Birkhoff. Dynamical systems with 2 degrees of freedom. Trans. Amer. Math. Soc. 18 (1917), 199-300.

[Bo] R. Bowen. Entropy and the fundamental group. Springer Lecture Notes in Maths 668, New York, 1978.

[FLP] A. Fathi, F. Laudenbach \& V. Poenaru, (eds). Travaux de Thurston sur les Surfaces. Asterisque 66-67 (1979).

[FS] A. Fathi \& M. Shub. Some dynamics of pseudo-Anosov diffeomorphisms. In [FLP], op. cit.

[Fr] J. Franks. Homology and Dynamical Systems. CBMS Reg. Conf. Series 49. Amer. Math. Soc.: Providence, 1982.

[F1] D. Fried. Geometry of cross-sections to flows. Topology 21 (1982), 353-371.

[F2] D. Fried. Fibrations with pseudo-Anosov monodromy. In [FLP], op. cit.

[F3] D. Fried. Flow equivalence, hyperbolic systems and a new zeta function for flows. Comm. Math. Helv. 57 (1982), 237-259.

[F4] D. Fried. Homological identities for closed orbits. Inv. Math. 71, 419-442 (1983).

[F5] D. Fried. Periodic points and twisted coefficients. In Geometric Dynamics, Proceedings of 1981 IMPA Symposium on Dynamical Systems. Springer Lecture Notes in Maths 1007, 261-293.

[F6] D. Fried. Entropy and twisted cohomology. Preprint.

[F7] D. Fried. Monodromy and dynamical systems. IHES preprint, July 1983.

[F8] D. Fried. Efficiency vs. hyperbolicity on tori. In Global Theory of Dynamical Systems, Springer Lecture Notes in Maths. 819.

[F9] D. Fried. Transitive Anosov flows and pseudo-Anosov maps. Topology, 22 (1983), 299-303.

[F10] D. Fried. Subshifts on surfaces. Ergod. Th. \& Dynam. Sys. 2 (1982), 15-21.

[I] G. Ikegami. Flow equivalence of diffeomorphisms. Osaka J. Math. 8 (1971), 49-70; 9 (1972), 335-336.

[L] W. Ljunggren. On the irreducibility of certain trinomials and quadrinomials. Math. Scand. 8 (1960), 65-70. 
[MKS] W. Magnus, A. Karass \& D. Solitar. Combinatorial Group Theory. Dover, 1971.

[M] J. Milnor. A duality theorem for Reidemeister torsion. Ann. Math. 76 (1962), 137-147.

[S] I. N. Sanov. A property of a representation of a free group. Doklady Akad. Nauk SSR 57 (1947), 657-659.

[T1] W. P. Thurston. On the geometry and dynamics of diffeomorphisms of surfaces I. Preprint.

[T2] W. P. Thurston. A norm for the homology of 3-manifolds. Preprint. 